\title{
„Ich sehe was, was du nicht siehst“
}

\section{Eine explorative Betrachtung verlaufsdiagnostischer Selbstbeurteilungen des Lernverhaltens}

\author{
Sina Napiany $\mathbb{0}$, Simone Weber, Christian Huber \\ Institut für Bildungsforschung, Bergische Universität Wuppertal, School of Education, Wuppertal
}

\begin{abstract}
Zusammenfassung: Hintergrund: Durch schulische Verhaltensverlaufsdiagnostik können Verhaltensveränderungen differenziert sichtbar gemacht und die Effektivität von Interventionsmaßnahmen überprüft werden. Erkenntnisse zu Gütekriterien von Direkter Verhaltensselbstbeurteilung $\left(D_{V} B_{\text {selbst }}\right)$, im Gegensatz zu Direkter Verhaltensbeurteilung als Fremdeinschätzung, liegen kaum vor. Das Hauptziel dieser explorativen Studie ist es, erste Hinweise zur Validität der DVB selbst in Hinblick auf das Lernverhalten von Schüler_innen aufzuzeigen. Außerdem soll untersucht werden, ob sich Verhaltensselbstbeurteilungen von Schüler_innen über die Zeit verändern. Methoden: Vier Wochen lang und dabei bis zu

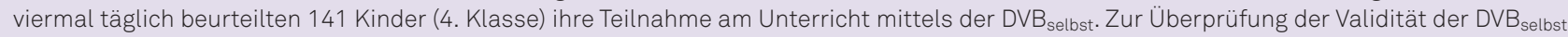
wurde das Lernverhalten zusätzlich mittels eines statusdiagnostischen standardisierten Fragebogens erhoben (Schülereinschätzliste für Sozial- und Lernverhalten/SSL). Ergebnisse: Die verlaufsdiagnostische Selbstbeurteilung des Lernverhaltens von Schüler_innen über eine Woche, gemessen mittels DVB $B_{\text {selbst }}$ steht im signifikanten Zusammenhang mit der Messung des Lernverhaltens mittels des statusdiagnostischen Fragebogens SSL. Die Selbstbeurteilung der Teilnahme am Unterricht mittels DVB Belbst unterscheidet sich signifikant zwischen Woche 1 und Woche 4.

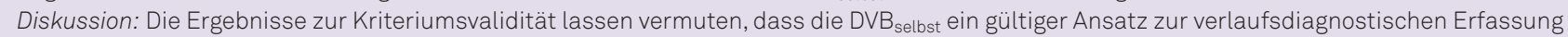
von Lernverhaltensweisen ist. Zudem zeigen die Resultate, dass sich Verhaltensselbstbeurteilungen von Schüler_innen über die Zeit ändern können. Die vorliegenden Ergebnisse sind mit Vorsicht zu interpretieren und sollten in zukünftigen Studien experimentell überprüft werden.
\end{abstract}

Schlüsselwörter: Verlaufsdiagnostik, ambulantes Assessment, Selbstbeurteilung, Lernverhalten, Validität

"I Spy With My Little Eye" - An Explorative Study to Investigate Frequent Self-Ratings of Learning Behavior

\begin{abstract}
Background: The developmental assessment of students' behavioral performance is considered to be a suitable tool to monitor taskand learning-related behavior changes and to evaluate intervention effects. However, and in contrast to respective observer assessments, the psychometric properties of direct behavior self-ratings $\left(\mathrm{DBR}_{\text {self }}\right)$ have been rarely investigated. Thus, the main aim of the present explorative study is to examine the validity of the DBR self for assessing students' learning behavior. Furthermore, we seek to investigate if students' selfratings might change over time. Methods: Upon using the $\mathrm{DBR}_{\text {self, }} 141$ fourth-grade students rated their academic engagement up to four times

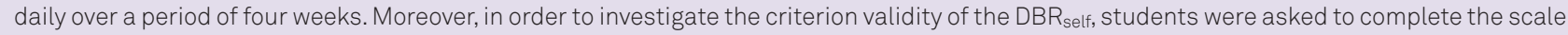
"learning behavior" of a standardized status diagnostic tool (i.e., Schülereinschätzliste für Sozial- und Lernverhalten / SSL). Results: Across a week, self-ratings of students' learning behaviors as measured by the process diagnostic tool DBR self $_{\text {and }}$ the status diagnostic questionnaire SSL are significantly correlated to each other. Moreover, students' self-ratings of academic engagement in week 1 differ significantly from those obtained in week 4. Discussion: Our findings suggest that the DBR self might be a valid tool to measure students' learning behavior and to monitor potential learning-related behavioral changes. Furthermore, our findings reveal that students' self-ratings of learning-related behaviors might change over time. The results of the present explorative study are preliminary and await further empirical support.
\end{abstract}

Keywords: Developmental behavioral assessment, ambulant assessment, self-monitoring, learning behavior, validity

\section{Einleitung}

Verlaufsdiagnostische Daten geben differenziert Auskunft, ob und wie sich individuelle Lern- und Verhaltensentwicklungen vollziehen und ob ausgewählte Förderungen wirksam sind (Casale, Hennemann \& Grosche 2015; Souvignier, 2018). Zur schulischen Verhaltensverlaufsdiagnostik beurteilen in der Regel Lehrkräfte mittels einer Direkten Verhaltensbeurteilung (DVB; engl. Direct Beha- vior Rating/DBR) hochfrequent ein ausgewähltes Lernoder Sozialverhalten von einzelnen Kindern (Casale et al., 2015).

Wird eine DVB als Selbstbeurteilung (DVB selbst $\left._{\text {s }}\right)$ durch Schüler_innen durchgeführt, ergeben sich weitere Vorteile. Unter anderem können mittels DVB selbst $_{\text {internali- }}$ sierende Verhaltensweisen wie beispielsweise soziale oder lernbezogene Ängstlichkeit diagnostisch erfasst werden. Außerdem bleiben die oft knappen Ressourcen 
einer Lehrkraft geschont. Eine theoretische Übersicht mit Blick auf die Vorteile eines solchen ambulanten Assessments bzw. Selbstmonitorings in der Schule liefern Bugl, Schmid und Gawrilow (2015).

Während die Güte von DVB in der Schule aus Fremdund Erwachsenenperspektive vergleichsweise gut evaluiert ist (Huber \& Rietz, 2015), ist eine DVB selbst $_{\text {weitest- }}$ gehend unerforscht. Erste empirische Ergebnisse zur Erfassung des Lernverhaltens mittels $\mathrm{DVB}_{\text {selbst }}$, bezüglich der Übereinstimmung von Lehrkraft-Schüler_innen Urteilen, konnten bereits aufgezeigt werden (Weber, Napiany \& Huber, 2020).

Ziel dieses Beitrags ist es, eine erste explorative Annäherung an die Validität einer schulischen DVB $_{\text {selbst }}$ mittels der Hinzunahme externer Kriterien der Selbstbeurteilung durchzuführen. Darüber hinaus wird ebenfalls explorativ betrachtet, ob bzw. wie sich Selbstbeurteilungen über die Zeit verändern.

\section{Verhaltensverlaufsdiagnostik in der Schule}

Eine Verhaltensverlaufsdiagnostik im Sinne der DVB stellt eine Kombination aus einer systematischen direkten Verhaltensbeobachtung und einer indirekten Verhaltensbeurteilung dar (Chafouleas, Kilgus \& Wallach, 2010; Christ, Riley Tillman \& Chafouleas, 2009). Eine festgelegte Verhaltensweise wird innerhalb eines Zeitraums, z.B. einer Unterrichtsstunde, in der Regel teilnehmend durch die Lehrkraft beobachtet. Unmittelbar im Anschluss wird dann deren Ausprägung eingeschätzt (Huber \& Rietz, 2015). Dies kann hochfrequent mehrmals täglich und über mehrere Wochen geschehen. Die gewonnenen Verlaufsdaten zeigen differenziert mögliche Veränderungen im Verhalten. Mittels einer DVB werden die Vorteile einer systematischen Verhaltensbeobachtung, die als verhaltensnächste Methode gilt (Schmidt-Atzert \& Amelang, 2012) und einer Verhaltensbeurteilung, die als ökonomisch gilt (Huber \& Rietz, 2015), kombiniert. Das zu beurteilende Verhalten sowie die Häufigkeit und Dauer der Beurteilungszeiträume kann nach Bedarf variiert werden (Chafouleas et al., 2010; Christ et al., 2009). So ist die Methode in unterschiedlichen schulischen Kontexten anwendbar und eine hohe ökologische Validität ist gegeben (Bugl et al., 2015). Flexibel ist eine DVB auch, da neben der üblichen Beurteilung aus Fremdperspektive eine Beurteilung aus Selbstperspektive denkbar ist. Weiterführende Informationen zur praktischen Umsetzung einer DVB bzw. des ambulanten Assessments in der Schule finden sich z.B. bei Casale, Huber, Hennemann und Grosche (2019) oder bei Bugl et al. (2015).

Um eine DVB, aus Fremd- oder Selbstperspektive, im Zuge von Verlaufsdiagnostik in der Schule praktisch durchzuführen und empirisch zu prüfen, ist es notwendig, dass a. eine passende Verhaltensweise zur Beobachtung festgelegt und

b. eine passende Antwortskala zur Beurteilung ausgewählt wird.

Passend ist eine Verhaltensweise im Zuge des vorliegenden Forschungsziels, wenn sie pädagogisch relevant ist, an wissenschaftliche Vorarbeiten anknüpft sowie gleichzeitig eine Validierung möglich ist. Passend ist eine Antwortskala, wenn diese kindgerecht ist und Verhaltensverläufe differenziert erfassen kann. Aus Erkenntnissen des bisherigen Forschungsstandes können Ableitungen für eine erste Überprüfung der Validität der DVB $_{\text {selbst }}$ getroffen werden.

\section{Ableitungen zur Auswahl einer Verhaltensweise}

Verhaltensverlaufsdiagnostik mittels DVB aus Fremd- und Erwachsenenperspektive war bereits Gegenstand zahlreicher Studien (eine Übersicht geben: Chafouleas, 2011; Huber \& Rietz, 2015).

Die sogenannten BIG 3 Verhaltensweisen der Teilnahme am Unterricht, des störenden Verhaltens und des respektvollen Verhaltens zur Beurteilung mittels DVB sind bereits mehrfach überprüft und hinsichtlich der Testgüte abgesichert (Briesch, Chafouleas \& Riley Tillman, 2010; Chafouleas, 2011; Huber \& Rietz, 2015). Diese Verhaltensweisen gelten als relevant für einen erfolgreichen Schulbesuch und können gut beobachtet werden (Chafouleas, 2011). In der Mehrzahl publizierter Studien zur DVB wurde die Teilnahme am Unterricht (engl. Academic Engagement) als Verhaltensziel ausgewählt und überprüft (Huber \& Rietz, 2015). Diese wird als aktive (z.B. Fragen beantworten, melden) und passive Beteiligung (z.B. zuhören, zuschauen) am Unterrichtsgeschehen definiert (Huber \& Rietz, 2015). Um eine ausgewählte Verhaltensweise zu beurteilen, werden für eine DVB Skala entweder ein Item (engl. Single-Item Scale) oder mehrere Items (engl. MultiItem Scale) ausgewählt (Huber \& Rietz, 2015). In der Mehrzahl publizierter Studien zur DVB wurden SingleItem Scales verwendet (Huber \& Rietz, 2015).

Auch Informationen aus dem Forschungsstand zu statusdiagnostischen schulischen Selbstbeurteilungen können die Auswahl einer passenden Verhaltensweise ergänzen. Diese Selbstbeurteilungen werden meist schriftlich durch standardisierte Fragebögen erfasst (Krüger, 2006). Hierbei werden Schüler_innen aufgefordert, anhand vorgegebener Aussagen (Items) einen bestimmten schulischen (Verhaltens-)Bereich retroperspektiv einzuschätzen. Diese Erinnerung und Beurteilung erfordert eine gewisse kognitive und metakognitive Reife und ist zudem, aufgrund möglicher Verzerrungen durch Erinnerungseffekte, fehleranfällig (Schmidt-Atzert \& Amelang, 2012). Sowohl die Differenziertheit von Selbstwahrnehmung und -beurteilung als auch die Lesekompetenz steigen mit zunehmender kognitiver 
Entwicklung (Neugebauer \& Wolter, 2018). Wenige Selbstbeurteilungsverfahren sind daher für Schüler_innen der ersten zwei Schulstufen konzipiert. Das Vorhandensein überprüfterstatusdiagnostischerVerfahrenzurSelbstbeurteilung des Sozial- oder Lernverhaltens im späten Grundschulalter zeigt, dass eine Selbstbeurteilung in diesem Altersbereich grundsätzlich valide ist. Lohbeck, Petermann und Petermann (2015) bestätigen in ihrer Studie die Validität von Beurteilungen des eigenen Lernverhaltens durch Schüler_innen im vierten Schuljahr. Diese Befunde gehen auch mit bisherigen Forschungen zum akademischen und sozialen Selbstkonzept einher, welches im Verlauf der Grundschulzeit weniger überschätzt und zeitgleich differenzierter wird (Harter, 2012; Hellmich \& Günther, 2011).

\section{Ableitungen zur Auswahl einer Antwortskala}

In bisherigen Forschungsarbeiten zur DVB wurden Antwortskalen sehr heterogen verwendet. Es wurden sowohl Skalen, die eine Intensitäts- als auch eine Häufigkeitsbeurteilung ermöglichen, eingesetzt als auch Skalen mit wenigen (z.B. sechs) und vielen (z. B. 14) Abstufungen. Chafouleas (2011) empfiehlt für eine DVB grundlegend den Einsatz einer 11-stufigen Antwortskala, um Veränderungen im Verlauf differenziert abbilden zu können.

Bei Selbstbeurteilungen durch Kinder zeigt sich, dass diese mit zunehmender kognitiver Reife seltener inadäquate oder unsichere (z.B. weiß nicht) Antworten geben (Emde \& Fuchs, 2012). Es lässt sich feststellen, dass für das Grundschulalter häufig Skalen im drei- bis sechsstufigen Bereich genutzt werden (z. B. Lohbeck, Schultheiß, Petermann \& Petermann, 2015; Rauer \& Schuck, 2003; Petermann \& Petermann, 2014). Bei der Verwendung einer vierstufigen Skala zeigt sich, dass Schüler_innen im Primarbereich ihr Sozial- und Lernverhalten statusdiagnostisch zwar überhöht positiv aber dennoch differenziert einschätzen (Lohbeck, Petermann et al., 2015).

\section{Zusammenfassung zur Umsetzung einer DVB selbst}

Der aktuelle Forschungsstand zur DVB und die Erkenntnisse aus statusdiagnostischen schulischen Selbstbeurteilungen sind jeweils nur eingeschränkt auf die $\mathrm{DVB}_{\text {selbst }}$ übertragbar.

Der Großteil der Studien zur DVB wurde unter Laborbedingungen und von Studierenden als Beurteiler_innen durchgeführt. Bei der vorliegenden Fragestellung verschiebt sich sowohl die Zugangsperspektive von einer Fremd- zu einer Selbstbeurteilung als auch der Entwicklungsstand der Beurteiler_innen. Außerdem handelt es sich um eine Überprüfung im Feld (d.h. direkt im schulischen Umfeld).

Statusdiagnostische Instrumente sind nicht für einen hochfrequenten Einsatz konzipiert und überprüft. Hieraus gewonnene Daten werden meist im interindividuellen
Vergleich und nicht im intraindividuellen Verlauf ausgewertet (Schmidt-Atzert \& Amelang, 2012). Bei statusdiagnostischen Selbstbeurteilungen zum Sozial- und Lernverhalten werden, im Gegensatz zur DVB selbst, $_{\text {, Verhal- }}$ tensweisen indirekt erfragt. Retrospektiv werden hierzu einzelne Lernverhaltensweisen zu einem Konstrukt auf globalerer Ebene zusammengefügt.

Zusammengefasst zeigt sich, dass Kinder im späten Grundschulalter eigene Verhaltensaspekte wahrnehmen und differenziert beurteilen können. Somit kann davon ausgegangen werden, dass eine DVB $_{\text {selbst }}$ von Lernverhaltensweisen und auch der Teilnahme am Unterricht durch Kinder in diesem Alter durchgeführt werden kann.

Die Auswahl der Teilnahme am Unterricht als Ziel für eine $\mathrm{DVB}_{\text {selbst }}$ schließt empirisch an nationale und internationale Forschungsarbeiten an. Auch aus theoretischer Sicht ist sie begründet. Am Unterricht teilzunehmen bedeutet, sich auf motivationaler, kognitiver und behavioraler Ebene auf diesen einzulassen (Fredricks, Blumenfeld, \& Paris, 2004). Dabei kann vielfach aufgezeigt werden, dass die Teilnahme am Unterricht in positivem Zusammenhang mit der Schulleistung steht (Alrashidi, Phan, \& Ngu, 2016). Alrashidi et al. (2016) geben vertiefend einen theoretischen Überblick zur Teilnahme am Unterricht. Praktisch ist diese Verhaltensweise somit für alle Schüler_innen gleichermaßen relevant. Eine Auswahl der Verhaltensweise der Teilnahme am Unterricht als Beurteilungsziel in einer breiten Stichprobe erscheint daher naheliegend.

Weiter lässt sich annehmen, dass es kindgerecht ist, eine Antwortskala von maximal sechs Stufen zu wählen. Die Befunde von Lohbeck, Petermann et al. (2015) zeigen, dass ein differenziertes Antwortverhalten grundsätzlich auch bei einer Skala mit wenigen Abstufungen erwartet werden kann. Eine Entscheidung zugunsten einer Skala mit oder ohne Mittelkategorie kann mittels einer Vorstudie überprüft werden.

\section{Güte einer DVB selbst $_{\text {in der Schule }}$}

In der schulischen Praxis ist das Konzept regelmäßiger Verhaltensselbstbeurteilungen nicht neu (Mooney, Ryan, Uhing, Reid \& Epstein, 2005). Erst seit kürzerer Zeit findet der Ansatz auch systematisch im Hinblick auf verlaufsdiagnostische Erhebung und Auswertung Beachtung (Bugl et al., 2015). Empirische Erkenntnisse zur Güte einer DVBselbst liegen, nach aktuellem Recherchestand, kaum vor.

Erste Ergebnisse zur Überprüfung der Reliabilität zeigen, dass im Mittel eine höhere Selbstbeurteilung der aktiven Teilnahme am Unterricht durch Kinder als bei einer Fremdbeurteilung durch Lehrkräfte anzunehmen ist (Weber et al., 2020). Für die Gesamtstichprobe konnten in dieser Studie weder auf numerischer noch auf struktur- 
eller Ebene zufriedenstellende Übereinstimmungen zwischen den Einschätzungen der Beurteilungspaare nachgewiesen werden. Auf fehlende Übereinstimmungen zwischen Urteilen von Lehrkräften und Schulkindern, insbesondere im Grundschulalter, weisen auch andere Forschungsarbeiten hin (Roos et al., 2016). So stellt sich die Frage, welche Möglichkeiten der Überprüfung der Güte einer $\mathrm{DVB}_{\text {selbst }}$ neben einer Lehrkraftbeurteilung ergänzend möglich sind, um den aktuellen Forschungsstand zu erweitern. Nach Schmidt-Atzert und Amelang (2012) kommen zur Validierung von Beobachtungs- und Beurteilungsverfahren unter bestimmten Bedingungen Korrelationen mit Fragebögen, Gruppenvergleiche sowie Veränderungsmessungen aufgrund bewährter Interventionen in Frage.

\section{Externe Validierungskriterien}

Um eine Annäherung an die Validität vorzunehmen, ist es möglich, einen Fragebogen, der das gleiche Merkmal erfasst (im vorliegenden Manuskript das Lernverhalten), hinzuzuziehen (Schmidt-Atzert \& Amelang, 2012). Beide methodischen Vorgehensweisen, eine DVB $_{\text {selbst }}$ und ein Fragebogen, messen Lernverhalten auf unterschiedlich spezifischen Ebenen. Durch eine DVB $_{\text {selbst }}$ wird ein Verhalten direkt im Kontext (und daher mit wenig Spielraum für Verzerrungen durch Interpretation) sowie zeitnah und somit sehr spezifisch erfasst. Ein Fragebogen misst einen Verhaltensbereich retroperspektiv und meist in Bezug auf einen globaleren Kontext. Eine Messung mit unterschiedlichem Kontextbezug stellt eine große Herausforderung, aufgrund des Prinzips der Spezifitätsanpassung, (engl. Specificity Matching Principle) dar (Swann, ChangSchneider, \& Larsen McClarty, 2007). Nach diesem Prinzip sollten Variablen bei Selbstbeurteilungen auf gleicher Spezifitätsebene erhoben werden, um möglichst viel Aufschluss über deren Beziehung zueinander zu erhalten (Swann et al., 2007). Durch die Messung zweier Variablen mit globalem Kontext (z. B. allgemeines Selbstwertgefühl \& allgemeine Lebenszufriedenheit) oder zweier Variablen mit spezifischem Kontext (z.B. Selbstkonzept in Mathematik \& Motivation in Mathematik) kann mehr Aufschluss erlangt werden als bei einer Messung auf unterschiedlichen Spezifitätsebenen (z.B. allgemeines Selbstwertgefühl \& Motivation in Mathematik). Eine Erfassung der Zusammenhänge von Variablen mit unterschiedlichen Spezifitätsebenen ist zwar möglich, liefert aber nur eine begrenzte Aufklärungsmöglichkeit.

Weiterführend ist es im Sinne der Kriteriumsvalidität möglich, stabile Forschungsbefunde, u. a. durch Gruppenvergleiche, als externe Kriterien heranzuziehen (SchmidtAtzert \& Amelang, 2012). Die bisherige Forschungslage bezüglich des Lernverhaltens weist darauf hin, dass geschlechtsspezifische Unterschiede wiederkehrende stabile Befunde sind. Ein Vergleich zeigt, dass sich Mädchen in fast allen Bereichen des schulischen Lernverhaltens und hinsichtlich ihres akademischen Selbstkonzepts höher einschätzen als Jungen (Petermann, Lohbeck \& Petermann, 2014; Weber \& Freund, 2017). Roos et al. (2016) konnten aufzeigen, dass sich Mädchen in Selbsteinschätzungen zusätzlich auch differenzierter und kongruenter einschätzen als Jungen.

\section{Verhaltensänderung durch Selbstbeobachtung und -beurteilung}

Verhaltensbeobachtungen durch externe Personen können zu einer Reaktanz bei der beobachteten Person und damit zu einer möglichen Änderung des Verhaltens führen (Schmidt-Atzert \& Amelang, 2012). Aus der Annahme heraus, dass auch eine Selbstbeobachtung zu Verhaltensänderungen führen kann, werden Selbstbeobachtungen und -beurteilungen als kognitive Interventionsansätze gezielt zur Verhaltensförderung eingesetzt (Schramm, 2018). Im Zuge dessen werden Selbstbeurteilungen meist mit Selbstoder Fremdverstärkungen verknüpft, unter anderem mit Feedback- und/oder Belohnungssystemen wie z.B. bei Menzies, Lane und Lee (2009) oder Moore, Prebble, Robertson, Waetford und Anderson (2001).

In einem systematischen Review fanden Briesch et al. (2019) lediglich 22 Einzelfallstudien (mit 81 Kindern der Primarstufe, meist mit sonderpädagogischen Förderbedarf), in denen Selbstbeurteilungen (selfmonitoring) der Teilnahme am Unterricht (on task behavior) unabhängig von einem weiteren Interventionsbaustein (z.B. Feedback) genutzt wurden. In diesem Übersichtsartikel wurde bezüglich einer Verhaltensänderung ein starker Effekt mittels phi-Koeffizienten gefunden $(\varphi=.83)$. Mehrmalige Selbstbeurteilungen führten zu einer Zunahme der Teilnahme am Unterricht. In jeder der 22 Studien fanden die Beurteilungen prompt statt (nach maximal 15 Minuten). Studien zu Verhaltensänderungen von mehrmaligen Selbstbeurteilungen nach 45 Minuten bzw. einer Schulstunde, wie es im Setting einer DVB üblich ist, liegen nach Briesch et al. (2019) nicht vor.

So stellt sich die Frage, inwieweit sich mehrmalige aufeinanderfolgende Selbstbeurteilungen, die immer nach einer Schulstunde stattfinden, im Laufe der Zeit verändern. Eine erste explorative Überprüfung dieser Frage kann ein Blick auf die Ausprägung einer $\mathrm{DVB}_{\text {selbst }}$ über die Zeit, insbesondere zu Beginn und am Ende eines festgelegten Zeitraums, zeigen.

\section{Fragestellungen und Hypothesen}

Nach aktuellem Recherchestand liegen bisher kaum Forschungskenntnisse zur Güte verlaufsdiagnostischer Verhaltensselbstbeurteilungen von Lernverhaltensweisen vor. 
Im Mittelpunkt dieser explorativen Studie steht zunächst die Fragstellung, inwieweit mit Hilfe eines inhaltlich nahestehenden Fragebogenverfahrens zum Lernverhalten und eines Gruppenvergleichs erste Hinweise zur Gültigkeit der Methode der $\mathrm{DVB}_{\text {selbst }}$ aufgezeigt werden können. Aufgrund bestehender Befunde zu Verhaltensänderungen durch Selbstbeobachtungen und -beurteilungen stellt sich außerdem die Frage, ob beim Einsatz der DVB selbst $_{\text {eine Ver- }}$ änderung im Verlauf der Zeit aufgezeigt werden kann. So leiten wir die folgenden Hypothesen zur Überprüfung ab:

\section{Hypothese 1}

Zwischen der Selbstbeurteilung des Lernverhaltens mittels eines standardisierten Fragebogens und der Lernverhaltensweisen mittels DVB $_{\text {selbst }}$, über den Zeitraum von einer Woche, besteht ein signifikanter Zusammenhang.

\section{Hypothese 2}

Mädchen beurteilen ihre Teilnahme am Unterricht mittels $\mathrm{DVB}_{\text {selbst }}$ insgesamt höher als Jungen.

\section{Hypothese 3}

Mädchen beurteilen ihre Teilnahme am Unterricht mittels $\mathrm{DVB}_{\text {selbst }}$ differenzierter und kongruenter als Jungen.

\section{Hypothese 4}

Die Selbstbeurteilungen der Teilnahme am Unterricht mittels DVB $_{\text {selbst }}$ unterscheiden sich signifikant im Verlauf der Erhebungswochen. In Woche 1 ist die Selbstbeurteilung der Teilnahme am Unterricht niedriger als in Woche 4.

\section{Methode}

\section{Stichprobe}

An der Studie nahmen insgesamt 183 Kinder der vierten Klasse teil. Nach Aufbereitung der Daten lagen vollständige Angaben von 141 Kindern (53\% Mädchen) vor. Diese stam- men aus acht Regelschulen in NRW. Die Kinder befanden sich zum Erhebungszeitpunkt in der Mitte des Schuljahres.

\section{Studienablauf und Design}

Die Durchführung der Erhebungen fand über einen Zeitraum von vier aufeinanderfolgenden Schulwochen statt. Hierzu wurden die von den Kindern wahrgenommene Teilnahme am Unterricht mehrmals täglich und das Lernverhalten am Ende einer Woche gemessen. Abbildung 1 gibt einen Überblick über den Studienablauf.

Viermal täglich, jeweils nach einer Schulstunde, sollte im Klassenverband eine DVB $_{\text {selbst }}$ stattfinden. Bei dieser Vorgabe konnten daher bis zu 20 Messungen wöchentlich und maximal 80 Messungen für den Gesamtzeitraum für jedes Kind vorliegen. Am Ende der zweiten Erhebungswoche wurde zusätzlich der statusdiagnostische standardisierte Fragebogen zum Lernverhalten eingesetzt. Die Schüler_innen beantworteten diesen Fragebogen einmalig in Bezug auf die vergangene Woche (Erhebungswoche 2). Die Durchführung des Fragebogens nach der zweiten Erhebungswoche war pragmatisch begründet. Die Kinder sollten genügend vertraut und damit sicher im Umgang mit der $\mathrm{DVB}_{\text {selbst }}$ sein. Die Instrumente wurden außerdem vorab in der Klasse durch geschulte Studierende eingeführt und von den Kindern erprobt.

\section{Instrumente}

DVB $_{\text {selbst }}$

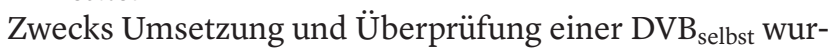
de die Lernverhaltensweise der Teilnahme am Unterricht ausgewählt. Um allen Kindern die Beurteilung der Verhaltensweise zu vermitteln, wurde eine kindgerechte Umformulierung zur Mitarbeit im Unterricht vorgenommen. Im Rahmen einer Einführung wurde mit den Kindern sowohl die passive (z.B. zuhören, zuschauen) als auch die aktive (z.B. Fragen beantworten, melden) Mitarbeit besprochen.

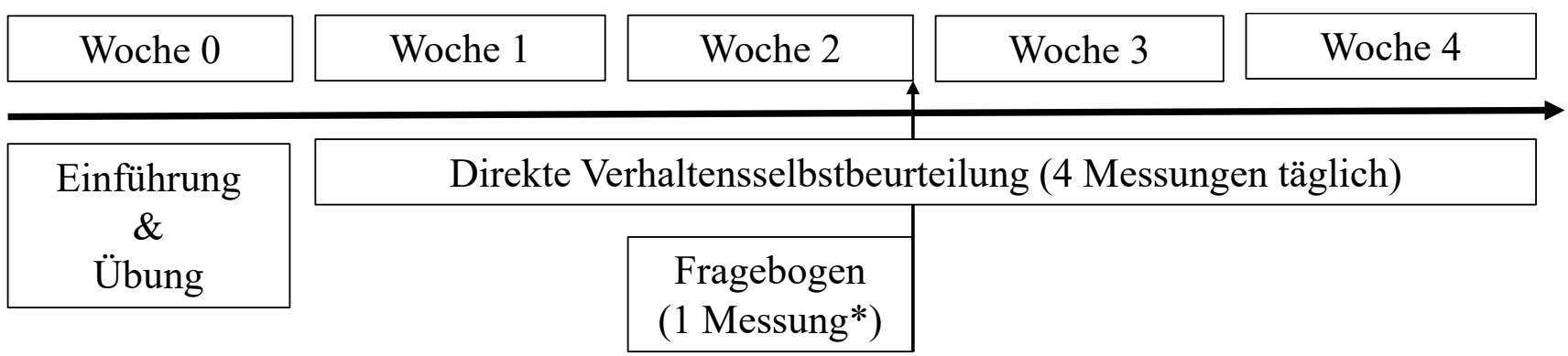

Abbildung 1. Studienablauf zur Selbstbeurteilung des Lernverhaltens im Verlauf. * Die Fragebogenerhebung fand am Ende von Woche 2 in Bezug auf die gesamte Woche statt. 
Zur Auswahl einer Antwortskala wurde auf eine kinderfreundliche fünf- bzw. sechsstufige Skala zurückgegriffen. In einer Vorstudie nutzten 48 Kinder beide Skalen für jeweils eine Woche. Die Kinder stimmten anschließend in Mehrheit für eine fünfstufige Skala und die Verwendung von Smileys, um die qualitativen Abstufungen zu symbolisieren. Diese fünfstufige Skala ( $\operatorname{Min}=1, \operatorname{Max}=5)$ wurde anschließend für die Hauptstudie verwendet.

Die Selbstbeurteilungen fanden appbasiert auf einem Tablet-PC statt und nahmen für die gesamte Klasse nur wenige Minuten in Anspruch. Die Kinder gingen nach der Unterrichtsstunde zu einem von zwei Tablet-PCs im Klassenraum. Sie wählten aus einer vorprogrammierten Liste ihren Vornamen aus und machten anschließend verdeckt ihre Angabe zur Teilnahme am Unterricht. Durch diese Umsetzung wurde die Wahrscheinlichkeit sozial erwünschter Antworten minimiert, da weder das Kind selbst auf vorherige Beurteilungen zurückgreifen konnte, noch andere Kinder oder die Lehrkraft Einsicht in die Selbstbeurteilung nehmen konnten.

\section{Fragebogen}

Zusätzlich wurde als statusdiagnostisches standardisiertes Instrument die Skala des Lernverhaltens der Schülereinschätzliste zum Sozial- und Lernverhalten (SSL) von Petermann und Petermann (2014) eingesetzt. Diese Skala mit 16 Items $(\alpha=.88)$ stellte zum Zeitpunkt der Studie das einzige inhaltlich nahestehende Konstrukt dar. Um dem Specificity Matching Principle zu folgen, wurde der Beurteilungszeitraum der Verhaltensweisen mittels SSL auf eine Woche festgelegt. So wurden die Kinder am Ende der Woche gebeten, ihr Lernverhalten nur in Bezug auf die vergangene Woche (Erhebungswoche 2) zu beurteilen. Nach Angabe des Manuals findet eine Beurteilung normalerweise in Bezug auf die letzten vier Wochen statt. Der Kontext zur Beurteilung des Verhaltens mittels Fragebogen wurde damit spezifischer.

Die Einschätzung erfolgte auf der vierstufigen Antwortskala (Min =0, Max =3), die die Auftretenshäufigkeiten von Verhaltensweisen abbildet (Verhalten tritt nie auf bis Verhalten tritt oft auf).

\section{Datenaufbereitung und Auswertung}

\section{Datenaufbereitung}

Analog zum Manual (Petermann \& Petermann, 2014) wurden nur Daten der SSL in die Auswertung einbezogen, die weniger als drei fehlende Werte enthielten. Zudem wurden nur Datensätze, bei denen in der SSL mehr als zweimal Antworten in zwei verschiedenen Antwortkategorien zu finden waren, in die Stichprobe aufgenommen. Es musste also bei der Beantwortung der SSL mindestens eine individuelle Spannweite (Range) von $R=1$ vorliegen. Bei 31 Fragebögen traf dies nicht zu. Bei Aufbereitung der Daten der $\mathrm{DVB}_{\text {selbst }}$ galt, dass im Minimum fünf Messungen pro Woche vorliegen mussten, um von einer Verlaufserhebung ausgehen zu können. Durch dieses Kriterium fielen $11 \mathrm{Da}-$ tensätze aus der Stichprobe. So liegen vollständige Datensätze von 141 Kindern vor.

\section{Datenauswertung}

Um die verlaufsdiagnostischen Daten mit den statusdiagnostischen Daten vergleichen zu können, werden diese zu Mittelwerten zusammengefasst. Im Zuge dieser Studie

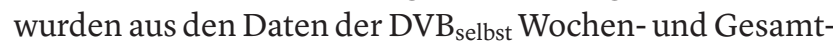
mittelwerte gebildet. Mittels Kolmogorov-Smirnoff-Tests wird die Normalverteilung der Daten betrachtet. Da keine Normalverteilung vorliegt, werden zur Auswertung der Hypothesen nonparametrische Verfahren gewählt.

Um die Kriteriumsvalidität über den Zusammenhang mit einem externen Instrument zu prüfen (Hypothese 1), werden einseitige Korrelationen nach Spearman berechnet. Hierzu werden die Mittelwerte der SSL Skala zum Lernverhalten bezüglich Erhebungswoche 2 und die Mittelwerte der 10 möglichen Messungen der Teilnahme am Unterricht mittels DVB selbst $_{\text {von Erhebungswoche } 2 \text { korre- }}$ liert.

Geschlechtsspezifische Unterschiede bei der DVB selbst werden zunächst mittels eines Mann-Whitney-U-Tests einseitig geprüft (Hypothese 2). Anschließend werden die Korrelationswerte zwischen SSL- und der DVB $_{\text {selbst }}$ im Hinblick auf mögliche Geschlechtsunterschiede und damit bezüglich der Kongruenz geprüft (Hypothese 3). Die Unterschiede zwischen den Korrelationen werden dazu nach Eid, Gollwitzer und Schmitt (2017) in einer einseitigen Testung berechnet. Um Aufschluss über die Differenziertheit im Antwortverhalten zu erhalten, werden explorativ die Antwortverteilung und die individuellen Spannweiten (Range) betrachtet. Ein möglicher Unterschied zwischen Jungen und Mädchen wird hierbei erneut mittels MannWhitney-U-Test geprüft (Hypothese 3).

Bezüglich möglicher Änderungen im Verlauf der Zeit (Hypothese 4) wird eine ANOVA mit Messwiederholung (Friedman-Test), die Unterschiede zwischen Messzeitpunkten aufzeigt, durchgeführt. Hierfür werden die Mittelwerte der vier Erhebungswochen verglichen. So wird zunächst ersichtlich, ob der Haupteffekt der Woche (Zeit) einen signifikanten Einfluss auf die Messung nimmt. Anschließend werden hypothesenbezogen einfache Kontraste berechnet. Über Kontraste können Einzelvergleiche hypothesenrelevanter Unterschiede und damit zwischen der ersten (Woche 1) und letzten (Woche 4) Erhebungswoche abgebildet werden (Döring \& Bortz, 2016). 


\section{Ergebnisse}

Tabelle 1 zeigt einen Überblick über die deskriptiven Werte der Stichprobe sowohl für die Daten der DVB selbst (Antwortskala 1-5) als auch des Fragebogens SSL (Antwortskala 0-3).

Insgesamt liegen 8572 Selbstbeurteilungen mittels $\mathrm{DVB}_{\text {selbst }}$ zur Teilnahme am Unterricht vor. Deskriptiv zeigt sich, dass sich die Antworten auf allen fünf möglichen Stufen verteilen. Es fallen deutlich mehr Antworten in die oberen Stufen und bilden damit eine linksschiefe Verteilung der Daten.

Tabelle 2 ist zu entnehmen, wie viele der 141 Kinder jeweils eine, zwei, drei, vier oder alle fünf Antwortkategorien mindestens einmal nutzen. Insgesamt nehmen knapp 90 Prozent der Kinder mindestens drei Abstufungen zur Beantwortung vor $(R=2-4)$. Der Median (Mdn) liegt als Zentralwert in der Mitte einer der Größe nach geordneten Datenreihe. In den vorliegenden individuellen Spannweiten liegt ein $M d n=4$ vor. Der Modalwert, als häufigster Wert der Spannweiten, liegt bei $D=3$. Ein KolmogorovSmirnoff-Test zeigt, dass die Verteilung der Spannweiten signifikant von einer Normalverteilung abweicht $(d(141)=$ $.23, p<.001)$. Die Mehrheit der Kinder zeigt eine hohe Spannweite im Antwortverhalten bei der $\mathrm{DVB}_{\text {selbst }}$.

Tabelle 2 sind vergleichsweise die individuellen Spannweiten bei der Beantwortung der SSL zu entnehmen. Von vier möglichen Abstufungen bei der Beantwortung der SSL nutzt der Großteil der teilnehmenden Kinder (74\%) zwei oder drei Antwortstufen.

\section{Zusammenhang der Messinstrumente}

\section{Hypothese 1}

Zunächst soll die Beziehung zwischen den Selbstbeurteilungen des Lernverhaltens mittels des Fragebogens SSL und der $\mathrm{DVB}_{\text {selbst }}$ über den Zeitraum von einer Woche im Hinblick auf einen signifikanten Zusammenhang überprüft werden. Die Befunde zeigen, dass das Lernverhalten, gemessen mittels SSL und die Teilnahme am Unterricht, gemessen mittels $\mathrm{DVB}_{\text {selbst }}$, zu einer zusammenhängenden Einschätzung der Ausprägung von Lernverhaltensweisen einer Woche kommen $\left(r_{s}=.36, p<.01, N=141\right)$. Hierbei handelt es sich um eine mittlere Effektstärke.

\section{Geschlechtsspezifische Befunde}

\section{Hypothese 2}

Weiterführend wird analysiert, ob sich bekannte Gruppenunterschiede aufgrund des Geschlechts in Bezug auf die Höhe der Beurteilungen mittels DVB selbst $_{\text {zugunsten von }}$ Mädchen finden lassen.

Tabelle 1. Deskriptive Daten der Gesamtstichprobe zur DVB selbst der Teilnahme am Unterricht (Antwortskala $1-5$ ) und des Fragebogens zum Lernverhalten (Antwortskala 0 - 3) differenziert nach Erhebungswochen

\begin{tabular}{lllllll}
\hline Instrument & Zeitraum & $M$ & $S D$ & $M_{\min }{ }^{a}$ & $M_{\max }{ }^{b}$ & $N$ \\
\hline DVB selbst & Woche 1 & 4.30 & 0.42 & 2.67 & 5 & 141 \\
& Woche 2 & 4.35 & 0.42 & 3.00 & 5 & 141 \\
& Woche 3 & 4.40 & 0.40 & 3.21 & 5 & 141 \\
& Woche 4 & 4.39 & 0.40 & 3.33 & 5 & 141 \\
& Woche 1-4 & 4.36 & 0.34 & 3.53 & 5 & 141 \\
\hline Fragebogen & Woche 2 & 2.46 & 0.39 & 0.88 & 2.94 & 141 \\
\hline
\end{tabular}

Anmerkungen: Beim Fragebogen handelt es sich um die Schülereinschätzliste zum Sozial- und Lernverhalten (SSL; Petermann \& Petermann, 2014). a zeigt den niedrigsten und ${ }^{b}$ den höchsten individuellen Mittelwert aller Kinder in der vorliegenden Stichprobe.

Tabelle 2. Anzahl der Kinder (in Prozent) mit individueller Spannweite (Range) im Antwortverhalten bei der DVB selbst und im Fragebogen

\begin{tabular}{|c|c|c|c|c|c|c|c|c|}
\hline & & $N$ & & & Range & & & \\
\hline & & & 0 & 1 & 2 & 3 & 4 & Gesamt \\
\hline \multirow[t]{3}{*}{ DVB selbst } & Mädchen & 74 & $1 \%$ & $11 \%$ & $46 \%$ & $27 \%$ & $15 \%$ & $100 \%$ \\
\hline & Jungen & 67 & $0 \%$ & $10 \%$ & $30 \%$ & $34 \%$ & $25 \%$ & $100 \%$ \\
\hline & Gesamt & 142 & $<1 \%$ & $11 \%$ & $38 \%$ & $31 \%$ & $20 \%$ & $100 \%$ \\
\hline Fragebogen & Gesamt & 173 & $18 \%^{a}$ & $44 \%$ & $30 \%$ & $7 \%$ & - & $100 \%$ \\
\hline
\end{tabular}

Anmerkungen: Beim Fragebogen handelt es sich um die Schülereinschätzliste zum Sozial- und Lernverhalten (SSL; Petermann \& Petermann, 2014). a Diese Datensätze wurden zur Analyse der Hypothesen ausgeschlossen (s. Datenaufbereitung). 
Mädchen erreichen einen Gesamtmittelwert ihrer DVBselbst Von $M=4.38$ ( $S D=0.31$ ), Jungen einen Gesamtmittelwert von $M=4.33(S D=0.36)$. Dieser Unterschied ist nicht signifikant $(U=2285.00, Z=-0.801, p=.21)$. Ein Blick auf mögliche Unterschiede in Erhebungswoche 2, zum Vergleich mit den Fragebogenangaben, zeigt ebenfalls keinen signifikanten Unterschied $(U=2371.00, Z=-0.45, p=.33)$ zwischen der Selbstbeurteilung der Mädchen $(M=4.34$, $S D=0.4)$ und Jungen $(M=4.36, S D=0.44)$.

Bei einer Betrachtung der Mittelwerte des Fragebogens SSL bezüglich Erhebungswoche 2 zeigt sich ein signifikanter Geschlechtsunterschied (Mädchen: $M=2.52, S D=0.35$; Jungen: $M=2.39, S D=0.42$ ). Die Mädchen bewerten ihr Lernverhalten in der Statusdiagnostik positiver bzw. höher als die Jungen $(U=1995.00, Z=-2.001, p<.05, d=.34)$. Es handelt sich um eine kleine Effektstärke.

\section{Hypothese 3}

Es wird analysiert, ob sich geschlechtsspezifische Unterschiede in Bezug auf die Kongruenz und die Differenziertheit bei der $\mathrm{DVB}_{\text {selbst }}$ zugunsten von Mädchen finden lassen.

Bezüglich der Kongruenz der Selbstbeurteilung zeigt eine Spearman-Rangkorrelation, dass das Lernverhalten, gemessen mittels Fragebogen SSL, und das Lernverhalten, gemessen mittels $\mathrm{DVB}_{\text {selbst }}$, bei Mädchen $\left(r_{s}=.43, p<.01\right.$, $N=74)$ in einem höheren Zusammenhang stehen als bei
Jungen $\left(r_{s}=.31, p<.01, N=67\right)$. Beide Korrelationen sind einzeln betrachtet signifikant und haben eine moderate bis hohe Effektstärke. Die Korrelationen unterscheiden sich nicht signifikant voneinander $(z=.81, p=.21)$. Abbildung 2 zeigt ein Streudiagramm und dazugehörige Trendlinien des Zusammenhangs von Selbsteinschätzungen des Lernverhaltens einer Woche mittels Fragebogen SSL und der $\mathrm{DVB}_{\text {selbst }}$, getrennt nach Geschlecht.

Um die Annahme zur differenzierteren Selbstbeurteilung von Mädchen zu betrachten, wird ein Blick auf das Antwortverhalten und die individuelle Spannweite bei der der $\mathrm{DVB}_{\text {selbst }}$ geworfen.

Deskriptiv zeigt sich, dass insgesamt sowohl Mädchen als auch Jungen die fünf verschiedenen Möglichkeiten der Antwortstufen im Kontext der DVB $_{\text {selbst }}$ nutzen. Ein geschlechtsspezifischer Unterschied der individuellen Spannweiten (Range) ( $U=2019.5, Z=-1.99, p<.05, d=-.03)$. Es handelt sich um einen schwachen Effekt.

\section{Zeitliche Veränderungen}

\section{Hypothese 4}

Abschließend wird überprüft, ob sich eine DVB $_{\text {selbst }}$ der Teilnahme am Unterricht im Laufe der Erhebungswochen unterscheidet bzw. in Woche 1 signifikant niedriger aus-

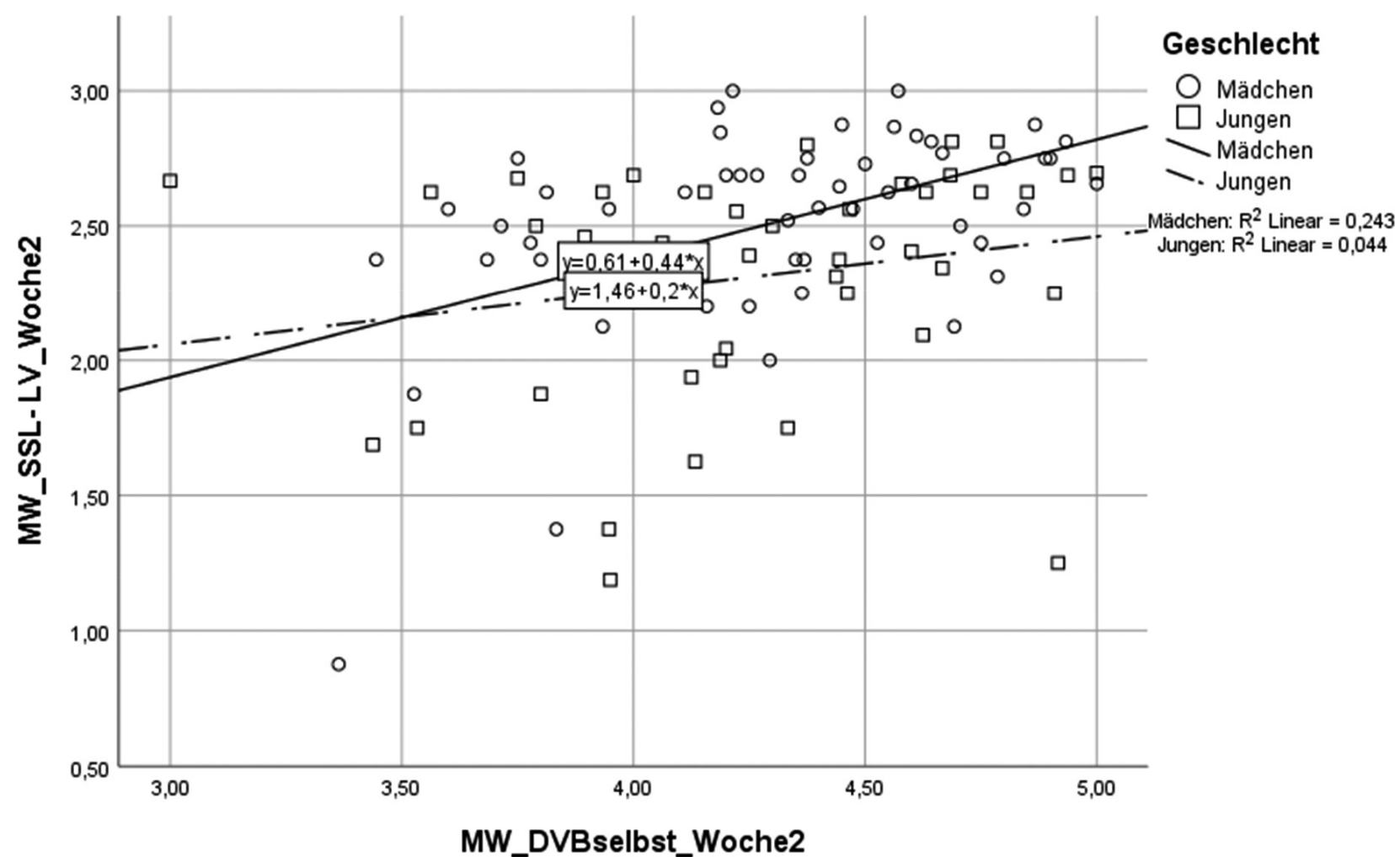

Abbildung 2. Streudiagramm und Trendlinien des Zusammenhangs von Selbsteinschätzungen des Lernverhaltens einer Woche mittels Fragebogen (SSL) und DVB selbst getrennt nach Geschlecht. 
fällt als in Woche 4. Eine Varianzanalyse mit Messwiederholung mit Huynh-Feldt Korrektur zeigt, dass sich die Teilnahme am Unterricht in Bezug auf den Hauptfaktor Wochen statistisch signifikant unterscheidet $(F(2.56$, $\left.358.28)=3.52, p<.05, \eta_{p}{ }^{2}=.03\right)$. Die Effektstärke ist als klein zu bezeichnen.

Bei der Durchführung eines einfachen Kontrastes zeigt sich ein signifikanter Unterschied zwischen Woche $1(M=4.3, S D=.04)$ und Woche $4(M=4.39, S D=.03)$ mit einer Mittelwertsdifferenz von 1.11 ( $S E=.21, p<.05$, partielles $\left.\eta_{p}{ }^{2}=.04\right)$. Die Effektstäke ist als klein zu bezeichnen.

\section{Diskussion}

Ziel der vorliegenden Studie war es, einen ersten explorativen Blick auf die Validität einer DVB $_{\text {selbst }}$ der Teilnahme am Unterricht zu werfen. Ergänzend wurde die Frage nach einer möglichen zeitlichen Veränderung der Teilnahme am Unterricht durch den Einsatz einer schulischen DVB $_{\text {selbst }}$ betrachtet.

Zunächst lassen sich aus den gewonnenen deskriptiven Erkenntnissen Rückschlüsse auf das methodische Vorgehen zur Umsetzung einer DVB $_{\text {selbst }}$ ziehen.

Die Auswahl der Verhaltensweise Teilnahme am Unterricht als Beurteilungsziel wurde aufgrund empirischer, theoretischer und praktischer Gründe vorgenommen. In der Einführung, Durchführung und Auswertung der Studie ließen sich keine Hinweise darauf finden, dass die teilnehmenden Kinder (der vierten Klasse) Probleme beim Verständnis und bei der Selbstbeurteilung dieser Verhaltensweise hatten.

Die Auswahl der fünfstufigen Antwortskala zeigte sich ebenfalls als treffend. Die Teilnahme am Unterricht mittels DVB $_{\text {selbst }}$ wurde zwar insgesamt sehr positiv, aber dennoch differenziert selbstbeurteilt. Von einigen Kindern wurden alle fünf Antwortkategorien genutzt. Die individuellen Spannweiten im Antwortverhalten lagen mit deutlichem Schwerpunkt im höheren Bereich.

\section{Einordnung hypothesenbezogener Ergebnisse}

\section{Zusammenhang der Messinstrumente}

Mangels Alternativen fiel die Auswahl eines passenden Außenkriteriums zur Überprüfung der Validität der $\mathrm{DVB}_{\text {selbst }}$ explorativ aus. Es wurde vermutet, dass die gemittelte Teilnahme am Unterricht in einer Erhebungswoche im mittleren Zusammenhang mit der statusdiagnostischen Einschätzung des Lernverhaltens ebendieser Woche steht. Dies konnte, trotz der unterschiedlichen Spezifitätsebenen der Instrumente, aufgezeigt werden. So lässt sich vermuten, dass der

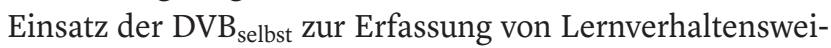
sen aus Selbstperspektive grundlegend möglich ist.

Eine Überprüfung des Zusammenhangs über einen Zeitraum von vier Wochen, wie dies dem regulären Einsatz der SSL entspricht, wäre hilfreich, um die vorliegenden explorativen Befunde bezüglich der Validität zu ergänzen.

\section{Geschlechtsspezifische Befunde}

Ziel war es anschließend, über Gruppenvergleiche geschlechtsspezifische Befunde bei der Selbstbeurteilung von Lernverhalten, zugunsten der Mädchen, zu prüfen. Es zeigte sich, dass Mädchen in der statusdiagnostischen Erhebung mittels des Fragebogens SSL ihr Lernverhalten signifikant höher selbstbeurteilen als Jungen. Dieses Ergebnis ist erwartungskonform $\mathrm{zu}$ anderen Erhebungen und den altersentsprechenden Normen der SSL (Petermann \& Petermann 2014; Roos et al., 2016). Der Geschlechtsunterschied zeigte sich in der gleichen Stichprobe, im Zuge der verlaufsdiagnostischen $\mathrm{DVB}_{\text {selbst }}$, jedoch nicht.

Auch der Zusammenhang zwischen den SSL Daten und den Daten der DVB $_{\text {selbst }}$ war bei beiden Geschlechtern in dieser Stichprobe ähnlich. Die mittlere verlaufsdiagnostische Einschätzung von Mädchen lag nicht näher an ihrer statusdiagnostischen Einschätzung im Vergleich mit Jungen. Unter Bedacht des explorativen Charakters dieser Studie, können die Selbstbeurteilungen von Mädchen und Jungen, als ähnlich kongruent bezeichnet werden.

Die Differenziertheit des Antwortverhaltens wurde durch die Betrachtung der individuellen Spannweiten untersucht. Hierbei zeigte sich ein signifikanter Geschlechtsunterschied, der jedoch als wenig bedeutsam einzuordnen ist. Zu beachten ist, dass Spannweiten generell anfällig für Ausreißer sind und daher keine Informationen über die Qualität dieser Differenziertheit abgeleitet werden können (Bortz \& Schuster, 2010).

Die geschlechtsspezifischen Befunde widersprechen insgesamt einigen Erkenntnissen zu statusdiagnostischen Selbsteinschätzungen des Lernverhaltens zugunsten von Mädchen (Lohbeck, Schultheiß et al., 2015). Das Geschlecht stellte damit im Zuge der vorliegenden Studie kein passendes Außenkriterium zur Überprüfung der Validität dar. Die angenommenen Unterschiede konnten mit den explorativen Auswertungsansätzen bei den verlaufsdiagnostischen Daten nicht aufgezeigt werden. Dies lässt sich inhaltlich in zwei Richtungen deuten. Einerseits könn-

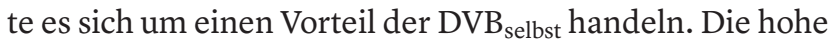
ökologische Validität und die zeitliche Nähe zum beurteilten Verhalten würden in dieser Sichtweise eventuell globale Störeinflüsse wie u.a. geschlechtsstereotype Repräsentationen, Erinnerungseffekte und andere Heuristiken reduzieren. Dies könnte nach Schramm (2018) durch die 
Entstehung von Wahrnehmungs- und Verhaltensdifferenzierungen erklärt werden. Andererseits könnte der Befund im Sinne einer verringerten Validität gedeutet werden. In diesem Fall würde mittels $\mathrm{DVB}_{\text {selbst }}$ Lernverhalten nicht entsprechend gängiger nahestehender Instrumente gemessen.

\section{Zeitliche Veränderungen}

Im Weiteren wurde der Hypothese nachgegangen, dass sich Beurteilungen mittels $\mathrm{DVB}_{\text {selbst }}$ über die Zeit verändern. Diese Annahme lag in Forschungsbefunden begründet, die aufzeigen, dass die Teilnahme am Unterricht im Verlauf regelmäßiger Selbstbeurteilungen höher bzw. besser wird (Briesch et al., 2019). Aufgrund des festgestellten kleinen Effekts in den vorliegenden Daten kann an dieser Stelle lediglich vermutet werden, dass eine Veränderung vorliegt.

\section{Limitationen}

Für eine Validierungsstudie ist die vorliegende Stichprobe als klein zu beschreiben, im Forschungsvorgehen von Einzelfallstudien durch eine DVB jedoch nicht. Außer dem Alter und Geschlecht sind keine weiteren Informationen über die Kinder sowie Variablen auf Klassenebene bekannt. Weitere Informationen über die Stichprobe wären wünschenswert (z.B. ob die Kinder bereits Erfahrungen mit Selbstbeurteilungen haben und welche Feedbackkultur oder Bezugsnormorientierung in den jeweiligen Klassen herrscht).

Im Zuge dieser Studie konnte lediglich die Teilnahme am Unterricht für die Anwendung mittels $\mathrm{DVB}_{\text {selbst }}$ betrachtet werden. Die Ergebnisse können nicht direkt auf weitere Verhaltensweisen übertragen werden. Eine Überprüfung der weiteren BIG 3s steht noch aus.

Bezüglich der Auswahl der Antwortskala stellt sich die Frage, ob Verhaltensveränderungen noch differenzierter hätten abgebildet werden können. Die Entscheidung für eine fünfstufige Skala im Vergleich zu einer elfstufigen Skala, wie sie sich im Kontext der DVB häufig bewährt hat (Briesch, Kilgus, Chafouleas, Riley-Tillman \& Christ, 2012), lag in dem Gedanken begründet, die Skala kindgerecht zu gestalten. Fuchs (2004) weist darauf hin, dass bei geringer kognitiver Reife anzunehmen ist, dass Antworten in Abhängigkeit der Antwortskala gegeben werden. In weiterführenden Studien wäre es relevant zu prüfen, ob eine Antwortskala mit breiterer Spanne zu einer differenzierteren Abbildung des Verlaufs beitragen kann. Dann würde auch linksschiefen Verteilungen bzw. Deckeneffekten entgegen gewirkt. Um Kinder in ihrem Verständnis der Antwortskala zu unterstützen, könnte ein ausführlicheres Training mit Feedback helfen. In der vorliegenden Studie gab es diesbezüglich lediglich eine Erklärung und eine Übung. Ein Training mit Feedback wird ebenfalls für den Einsatz der DVB durch Lehrkräfte empfohlen (Schlientz, Riley-Tillman, Briesch, Walcott \& Chafouleas, 2009).

Insgesamt gilt es zu bedenken, dass alle Auswertungsansätze explorativ gewählt wurden. So wurden lediglich zwei Außenkriterien zur Überprüfung der Validität herangezogen. Die Ergebnisse bezüglich der Validität sind daher nur als erste Hinweise zu betrachten. Zur weiteren Annäherung an die Validität könnten zusätzliche statusdiagnostische Instrumente beitragen. Möglich wäre es auch, eine systematische Fremdbeobachtung bzw. -beurteilung durch andere Kinder heranzuziehen. So würde das Verhalten auf gleicher Spezifitätsebene gemessen. Darüber hinaus wäre zur weiteren Überprüfung auch die Hinzunahme von Schulnoten möglich, wenn die $\mathrm{DVB}_{\text {selbst }}$ auch fachspezifisch ausgewertet würde.

Um die Annahme der zeitlich bedingten Veränderungen aufgrund regelmäßiger Selbstbeurteilungen mittels DVBselbst zu überprüfen, ist das Vorgehen in der vorliegenden Studie ebenfalls nur explorativ geeignet. Im Zuge dieser Studie handelt es sich lediglich um ein Innersubjekt Design zu mehreren Messzeitpunkten. Zusätzlich ist auch der Zeitraum von vier Wochen verhältnismäßig kurz, insbesondere, da bei einer Feldstudie häufig Messzeitpunkte ausfallen. Eine Studie mit Kontrollgruppe und über einen längeren Zeitraum wäre notwendig, um die Fragestellungen aussagekräftig zu beantworten. Sollte sich eine Verhaltensänderung in einem experimentellen Design aufzeigen lassen, hätte dies eine hohe praktische Relevanz. Regel-

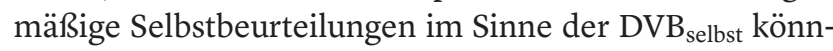
ten dann präventiv zur allgemeinen Förderung von positiven Verhaltensweisen im Unterricht und ggf. klassenweit eingesetzt werden. Hierbei würden die Ressourcen der Lehrkraft nicht bedeutend belastet.

\section{Relevanz für die Praxis}

Ein kleiner Beitrag zur Überprüfung der Gütekriterien der $\mathrm{DVB}_{\text {selbst }}$ in der Schule konnte mit dieser Studie erbracht werden. Auch wenn der Weg der wissenschaftlichen Absicherung der Methode der DVB $_{\text {selbst }}$ noch lang ist, zeigen die Ergebnisse dieser explorativen Studie auf, dass die

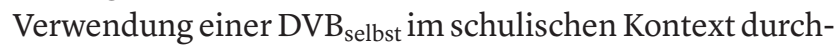
aus informativ ist. Auch in Hinblick auf die Gütekriterien einer $\mathrm{DVB}_{\text {selbst }}$ sind die vorliegenden Ergebnisse vielversprechend. Nicht nur als Baustein einer Intervention auf Einzelfallebene, so wie dies aktuell Anwendung findet, sondern auch zu verlaufsdiagnostischen Zwecken könnte die Methode der DVB $_{\text {selbst }}$ aufgrund ihrer Vorteile vermehrt Einzug in Schule und Unterricht finden. Eine Beurteilung aus Selbst- und Fremdperspektive eröffnet 
Gesprächsanlässe, stärkt die Mitbestimmung und Compliance des Kindes und eröffnet einen Zugang zu internalisierenden Problemen. Die Vorteile der Nutzung verlaufsdiagnostischer Daten zur Ableitung von Verhaltensänderungen und der Wirkung von eingeführten Interventionen liegen auf der Hand. Diese Daten können eine wichtige Ergänzung zur Nutzung statusdiagnostischer Befunde darstellen.

Die Praxistauglichkeit einer $\mathrm{DVB}_{\text {selbst }}$ ist insbesondere dann gegeben, wenn eine Durchführung und Auswertung ökonomisch (z.B. über digitale Hilfsmittel) möglich sind. Auch in diesem Bereich wären Weiterentwicklungen und wissenschaftliche Überprüfungen wünschenswert.

\section{Literatur}

Alrashidi, o., Phan, H. \& Ngu, B. (2016). Academic Engagement: An Overview of Its Definitions, Dimensions, and Major Conceptualisations. International Education Studies, 9(12), pp.41-52. doi:10.5539/ies.v9n12p41

Bortz, J. \& Schuster, C. (2010). Statistik für Human-und Sozialwissenschaftler. Berlin: Springer.

Briesch, A. M., Chafouleas, S. M. \& Riley Tillman, T. C. (2010). Generalizability and dependability of bahavior assessment methodes to estimate academic engagement: A comparison of systematic direct observation and direct behavior rating. School Psychology Review, 39, pp. $408-421$.

Briesch, A. M., Kilgus, S. P., Chafouleas, S. M., Riley-Tillman, T. C. \& Christ, T.J. (2012). The influence of alternative scale formats on the generalizability of data obtained from direct behavior rating single-item scales (DBR-SIS). Assessment for effective intervention. 38(2), pp. 127 - 133. doi:10.1177/1534508412441966

Briesch, A., Daniels, B. \& Beneville, M. (2019). Unpacking the Term "Self-Management": Understanding Intervention Applications Within the School-Based Literature. Journal of Behavioral Education, 28, pp. 54-78. doi:10.1007/s10864-018-9303-1

Bugl, P., Schmid, J. \& Gawrilow, C. (2015). Ambulantes Assessment in der Schule: Den schulischen Alltag erfahrbar machen. Lernen und Lernstörungen, 4, S.261-268. doi:10.1024/2235-0977/ a000115

Casale, G., Hennemann, T. \& Grosche, M. (2015). Zum Beitrag der Verlaufsdiagnostik für eine evidenzbasierte sonderpädagogische Praxis am Beispiel des Förderschwerpunkts der emotionalen und sozialen Entwicklung. Zeitschrift für Heilpädagogik, 66(7), S. $325-334$.

Casale, G., Huber, C., Hennemann, T. \& Grosche, M. (2019). Direkte Verhaltensbeurteilung in der Schule. München: Ernst Reinhardt.

Chafouleas, S. (2011). Direct behavior rating: a review of the issues and research in its development. Education and Treatment of Children, 34(4), pp. 575-591. Retrieved from https://www.jstor. org/stable/42900135

Chafouleas, S., Kilgus, S. \& Wallach, N. (2010). Ethical dilemmas in school-based behavioral screening. Assessment for Effective Intervention, 35(4), pp. 245 - 252. doi:10.1177/1534508410379002

Christ, T., Riley Tillman, T. \& Chafouleas, S. (2009). Foundation for the development and use of direct behavior rating (DBR) to assess and evaluate student behavior. Assessment for Effective Internvetion, 34(4), pp. 201 - 213. doi:10.1177/1534508409340390
Döring, N. \& Bortz, J. (2016). Forschungsmethoden und Evaluation in den Sozial- und Humanwissenschaften (5 Ausg.). Berlin: Springer.

Eid, M., Gollwitzer, M. \& Schmitt, M. (2017). Statistik und Forschungsmethoden. Weinheim: Beltz.

Emde, M., \& Fuchs, M. (2012). Datenqualität in standardisierten Interviews mit Kindern. In F. Heinzel (Hrsg.), Methoden der Kindheitsforschung. Ein Überblick über Forschungszugänge der kindlichen Perspektive (2. überarb. Ausg., S. 335 -349). Weinheim: Beltz.

Fredricks, J. A., Blumenfeld, P. C. \& Paris, A. H. (2004). School Engagement: Potential of the Concept, State of the Evidence. Review of Educational Research, 74(1), pp.59-109. doi:10.3102/ 00346543074001059

Fuchs, M. (2004). Kinder und Jugendliche als Befragte: Feldexperimente zum Antwortverhalten Minderjähriger. 28(54), S. 60 - 88. Von https://nbn-resolving.org/urn:nbn:de:0168-ssoar-207664 abgerufen

Harter, S. (2012). The Construction of the Self: Developmental and Sociocultural Foundations (2 ed.). New York: Guilford Press.

Hellmich, F. \& Günther, F. (2011). Entwicklung von Selbstkonzepten bei Kindern im Grundschulalter - ein Überblick. In F. Hellmich (Hrsg.), Selbstkonzepte im Grundschulalter (S.19-46). Stuttgart: Kohlhammer.

Huber, C., \& Rietz, C. (2015). Direct Behavior Rating (DBR) als Methode zur Verhaltensverlaufsdiagnostik in der Schule: Ein systematisches Review von Methodenstudien. Empirische Sonderpädagogik, 7(2), S. $75-98$.

Krüger, H.-H. (2006). Forschungsmethoden in der Kindheitsforschung. Diskurs Kindheits- und Jugenforschung, 1(1), S. 91 - 115. Von http://nbn-resolving.de/urn:nbn:de:0111-opus-9874 abgerufen

Lohbeck, A., Petermann, F. \& Petermann, U. (2015a). Selbsteinschätzungen zum Sozial- und Lernverhalten von Grundschulkindern der vierten Jahrgangsstufe. Zeitschrift für Entwicklungspsychologie und pädagogische Psychologie, 47(1), S. 1-13. doi:10.1026/0049-8637/a000118

Lohbeck, A., Schultheiß, J., Petermann, F. \& Petermann, U. (2015b). Die deutsche Selbstbeurteilungsversion des Strengths and Difficulties Questionnaire (SDQ-Deu-S). Diagnostica, 61(4), S. $222-235$.

Menzies, H. M., Lane, K. L. \& Lee, J. M. (2009). Self-monitoring strategies for use in the classroom: a promising practice to support productive behavior for students with emotional or behavioral disorders. Beyond Behavior, 18(2), pp. $27-35$.

Mooney, P., Ryan, J., Uhing, B., Reid, R. \& Epstein, M. (2005). A Review of Self-Management Interventions Targeting Academic Outcomes for Students with Emotional and Behavioral Disorders. Journal of Behavioral Education, 14, pp. 203-221. doi:10.1007/s10864-005-6298-1

Moore, D. W., Prebble, S., Robertson, J., Waetford, R. \& Anderson, A. (2001). Self-recording with goal setting: a self-management programme for the classroom. Educational Psychology, 21(3), pp. 255 - 265. doi:10.1080/01443410120065469

Neugebauer, R. \& Wolter, P. (2018). Selbstauskunft im Fluss: Die Entwicklungsperspektive. Kindheit. In W. Greve, Das Selbst. Psychologische Perspektiven (S. 78 - 97). Hildesheim: Universitätsverlag Hildesheim.

Petermann, F., Lohbeck, A. \& Petermann, U. (2014). Geschlechtsunterschiede im selbst eingeschätzten Sozial- und Lernverhalten und in den Mathematik- und Deutschnoten von Schülern. Zeitschrift für Soziologie der Erziehung und Sozialisation(4), S. 405 - 421.

Petermann, U. \& Petermann, F. (2014). SSL. Schülereinschätzliste für Sozial- und Lernverhalten. Göttingen: Hogrefe.

Rauer, W. \& Schuck, K. (2003). Fragebogen zur Erfassung emotionaler und sozialer Schulerfahrungen von Grundschulkindern dritter und vierter Klassen. Göttingen: Beltz. 
Roos, S., Lohbeck, A., Petermann, F., Petermann, U., Schultheiß, J., Nitkowski, D. et al. (2016). Fremd- und Selbsturteile von Lehrern und Schülern im Rahmen psychologischer Diagnostik. 64(3), S. 199 -207. doi:10.1024/1661-4747/a000279

Schlientz, M., Riley-Tillman, T., Briesch, A., Walcott, C. \& Chafouleas, S. (2009). The impact of training on the accuracy of direct behavior ratings (DBR). 24(2), pp. 73 - 83. doi:10.1037/a0016255

Schmidt-Atzert, L. \& Amelang, M. (2012). Psychologische Diagnostik (5. überarb. und erw. Ausg.). Berlin: Springer.

Schramm, S. (2018). Selbstmanagement-Therapie. In G. Lauth, \& F. Linderkamp (Hrsg.), Verhaltenstherapie mit Kindern und Jugendlichen (4. voll. überarb. Ausg., S.696-705). Weinheim: Beltz.

Souvignier, E. (2018). Computerbasierte Lernverlaufsdiagnostik. Lernen und Lernstörungen, 7, S. 219-223. doi:10.1024/22350977/a000240

Swann, W. B., Chang-Schneider, C. \& Larsen McClarty, K. (2007). Do people's self-views matter? Self-concept and self-esteem in everyday life. American Psychologist, 62(2), pp. 84-94.

Weber, K. E., \& Freund, P. A. (2017). Erfassung des Selbstkonzepts von Kindern im Grundschulalter. Zeitschrift für Entwicklungspsychologie und Pädagogische Psychologie, 49(1), S.38-49. doi:10.1026/0049-8637/a000165

Weber, S., Napiany, S. \& Huber, C. (2020). Können Schülerinnen und Schüler ihr Lernverhalten im Verlauf zuverlässig selbst beurteilen? Empirische Sonderpädagogik, 12(3), S. $207-222$.

\section{Historie}

Manuskript eingereicht: 28.10.2020

Manuskript angenommen: 29.04.2021

Onlineveröffentlichung: 25.05.2021

\section{Förderung}

Open-Access-Publikation ermöglicht durch die Bergische Universität Wuppertal.

\section{ORCID}

Sina Napiany

(D) https://orcid.org/0000-0003-3169-3180

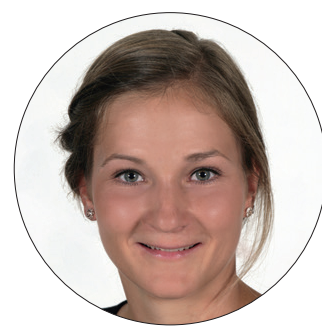

\section{Sina Napiany}

Institut für Bildungsforschung

Bergische Universität Wuppertal,

School of Education

Gaußstraße 20

42119 Wuppertal

Deutschland

napiany@uni-wuppertal.de 